\title{
Moduli of certain Fano 4-folds
}

Walter L. Baily, Jr.

Dedicated to the Memory of Professor Kunihiko Kodaira

\begin{abstract}
In this brief note we give a proof that a certain family of Fano 4-folds, described below, is complex (locally) complete and effectively parametrized in the sense of Kodaira-Spencer [Ko-Sp].
\end{abstract}

In this note we consider a specific family of Fano 4-folds which is analogous in some ways to the family of nodal Enriques surfaces, considered in my article which appeared in the March, 2000, issue of the Asian Journal of Mathematics, dedicated to the late Professor Kunihiko Kodaira. This is all part of a program intended to investigate certain moduli problems of a very special nature related to trying to find some family of algebraic varieties whose moduli would be related to the exceptional 27-dimensional domain associated to the real form $E_{7(-25)}$ of the exceptional Lie group $E_{7}$. In this note we settle a conjecture left open in my Asian Journal article. Numerous authors have considered Fano 3-folds and their classification, notably Iskovskikh, Kollar, Mori, and others. Little has been known about Fano 4-folds, and the current article is intended to break some new ground here. Actually I strongly suspect that the methods used here could be used to investigate a much wider family of Fano varieties, and suggesting such possibilities is one of its aims. The immediate background of the current note is to extend the results of the Asian Journal article and also of my article [AA] in the volume "Algebra and Analysis", Eds. Arslanov, Parshin, and Shafarevich, dedicated to N. G. Chebotarev.

At this point we shall state the main result of this note. In it we prove that a certain family of Fano 4-folds, to be described below, is everywhere locally complex-analytically complete and effectively 
parametrized. At this point we can say little about whether these Fano 4 -folds have a global complex moduli space. This still seems to be a difficult problem. But we believe that the main result here is new and rather interesting. In achieving it we have had a lot of help from Dr. Brendan Hassett, now at the Chinese University of Hong Kong, Professor Yujiro Kawamata of Tokyo University, and Professor Thomas Peternell of the University of Bayreuth. We acknowledge this help gratefully.

Notation. Let $Y=G(2,6)=\mathbf{G}(1,5)$, the variety of planes in the 6 -dimensional linear space or the variety of projective lines $\mathbf{P}^{1}$ in $\mathbf{P}^{5}$.

We embed $Y$ into $\mathbf{P}^{14}$ by Plücker coordinates. Let $Q$ be, at first, a generic quadric hypersurface in $\mathbf{P}^{14}$ and $\mathbf{P}^{11}$ be a generic linear subspace of codimension 3 in $\mathbf{P}^{14}$. Thus, $\mathcal{Q}=\mathbf{P}^{11} \cap Q$ is a generic quadric of codimension 4 in $\mathbf{P}^{11}$, while $\operatorname{dim} Y=8$; therefore $X=Y \cap \mathcal{Q}$ is a smooth variety of dimension 4 , and the canonical class $K_{X}$ of $X$ is $\{-L\}$, where $L$ is a hyperplane section of $X$; thus, the anti-canonical class of $X$ is very ample. Moreover, $X$ is easily seen to be simplyconnected. Therefore $X$ is a Fano 4-fold and is clearly of index 1, and $\operatorname{Pic}(X)=\mathbf{Z} \cdot L$. Let $T_{X}$ be the sheaf of germs of holomorphic crosssections of the holomorphic tangent bundle on $X$.

We shall prove that the family of all $X=Q \cap \mathbf{P}^{11} \cap Y$, assuming the intersection to be proper, is complete and effectively parametrized. To achieve this, it is sufficient by Kodaira and Spencer's criterion [Ko$\mathrm{Sp}],[\mathrm{Ko}]$ to show that

$$
H^{2}\left(X, T_{X}\right)=\{0\}
$$

and

$$
H^{0}\left(X, T_{X}\right)=\{0\} .
$$

Thanks to a communication from Prof. T. Peternell, the vanishing of $H^{2}\left(X, T_{X}\right)$ can be seen as follows: From Serre duality one has $(n=4)$

$$
H^{2}\left(X, T_{X}\right) \cong H^{n-2}\left(X, \Omega_{X}^{1} \otimes K_{X}\right)
$$

Now apply the vanishing criterion of Kodaira-Akizuki-Nakano for $L=$ $-K_{X}$ : If $L$ is ample on $X=X^{(n)}$, then

$$
H^{(p, q)}\left(X, L^{*}\right)=\{0\},
$$

for $p+q<n$. (Here $n=4$ and $L^{*}$ is the sheaf dual to $L$, namely $L^{*}=\Omega_{X}^{1} \otimes(-L)$.) 
Thus it remains to prove that $H^{0}\left(X, T_{X}\right)=\{0\}$. Henceforth, if $A$ is a coherent analytic sheaf on $X$, we shall write simply $H^{k}(A)=$ $H^{k}(X, A)$.

First we make a calculation of the number of independent complex parameters needed to describe $X$ as a subvariety of $\mathbf{P}^{14}$. We can easily see that the space of codimension 3 subspaces $\mathbf{P}^{11}$ has dimension equal to

$$
\operatorname{dim} G(11,14)=(11+1)(14-11)=36 .
$$

The dimension of the space of homogeneous quadrics in $\mathbf{P}^{14}$ is equal to the dimension of the space of $15 \times 15$ symmetric matrices $=120$; this is the linear homogeneous dimension. The ideal of quadric relations among the Plücker coordinates, i.e., the ideal of quadric relations vanishing on $\mathbf{G}(1,5)$, has dimension 15 . The dimension of the space of quadrics belonging to the ideal generated by 3 linear forms, i.e., the dimension of the space of quadrics vanishing on $\mathbf{P}^{11}$, is 42 . Moreover, $\operatorname{dim}(\operatorname{Aut}(G(2,6)))=\operatorname{dim} P G L(6)=35$. Then the number of effective parameters to describe $X$ as a subvariety of $\mathbf{P}^{14}$ is $36+120-15-42-1-35=63$, where we have subtracted 1 to account for the change from linear to projective coordinates in calculating the number of quadrics in $\mathbf{P}^{14}$.

It has been shown by O. Küchle [Ku2], and by Borcea [Bor2], that every sufficiently small deformation of $X$ is described by the same type of equations as a subvariety of $\mathbf{G}(1,5)$. In fact, $X$ can be described as the projection on $Y=G(2,6)$ of the set of zeros of a cross-section of a rank 4 vector bundle $E$ over $Y$. Specifically, according to [Bor2], let $H$ be a smooth irreducible divisor on $Y$ which generates $\mathrm{Pic}(Y)$, let $d_{1}=d_{2}=d_{3}=1$ and $d_{4}=2$, let $E$ be the rank 4 vector bundle on $Y$ given by

$$
E=[H] \oplus[H] \oplus[H] \oplus[2 H]
$$

where $[\mathrm{D}]$ is the complex line bundle over $Y$ associated to the divisor $D$. Then $X$ belongs to the family $\mathcal{F}$ of smooth, global complete intersections in $Y$, parametrized by the open set of $H^{0}(Y, E)$ consisting of sections of $E$ transversal to the zero-section. According to [Bor2, Theorem], $\mathcal{F}$ is complex-analytically complete, and every small deformation of $X$ is contained in $Y$ and is obtained by a small change in the crosssection defining $X$; i.e., by small changes in $\mathbf{P}^{11}$ and in $\mathcal{Q}$, modulo the relations described above. Since $H^{2}\left(T_{X}\right)=\{0\}$, it is well known that the versal deformation space $V_{X}$ of $X$ is smooth, has dimension equal to $\operatorname{dim} H^{1}\left(T_{X}\right)$, and by the above calculations, this dimension is 63 . 
Now the calculation of $h^{0}\left(T_{X}\right)$ is based on the three articles by Küchle, $[\mathrm{Ku} 1,2,3]$, numerous communications and conversations with Brendan Hassett, email correspondence with Prof. T. Peternell, and conversations with Y. Kawamata.

Brendan Hassett has noted the following facts. In order to compute

$$
H^{i}\left(\mathrm{Gr}(2,6), I_{X} \otimes T_{\mathrm{Gr}(2,6)}\right)
$$

we take the Koszul resolution of $I_{X}$, the homogeneous ideal of $X$ in $Y=\operatorname{Gr}(2,6)$, which is

$$
\begin{aligned}
0 \longrightarrow \mathcal{O}_{\mathrm{Gr}}(-5) \longrightarrow \mathcal{O}_{\mathrm{Gr}}(-4)^{\oplus 3} \oplus \mathcal{O}_{\mathrm{Gr}}(-3) \\
\longrightarrow \mathcal{O}_{\mathrm{Gr}}(-2)^{\oplus 3} \oplus \mathcal{O}_{\mathrm{Gr}}(-3)^{\oplus 3} \longrightarrow \mathcal{O}_{\mathrm{Gr}}(-1)^{\oplus 3} \oplus \mathcal{O}_{\mathrm{Gr}}(-2) \\
\longrightarrow I_{X} \longrightarrow 0
\end{aligned}
$$

$\mathcal{O}_{\text {Gr }}$ being the structural sheaf on Gr. By examining the roots and simple roots of $S L_{6}$ and applying Bott's Theorem IV (see [Bot]), we obtain

$$
H^{i}\left(\mathrm{Gr}, T_{\mathrm{Gr}}(-1)\right)=0
$$

for all $i$ and

$$
H^{i}\left(\mathrm{Gr}, T_{\mathrm{Gr}}(-n)\right)=0
$$

for $i<8$ and $n>1$. Since $T_{\mathrm{Gr}}$, the holomorphic tangent bundle to $\mathrm{Gr}$, is a locally free sheaf, we can tensor the above resolution by it to obtain another exact sequence which implies, finally, that

$$
H^{i}\left(\operatorname{Gr}(2,6), I_{X} \otimes T_{Y}\right)=0, \quad i=0,1 .
$$

Now one has the exact sequence

$$
0 \longrightarrow T_{X} \longrightarrow T_{Y} \mid X \longrightarrow N_{X} \longrightarrow 0
$$

where $N_{X}$ is the normal bundle to $X$ in $Y$, and

$$
N_{X} \cong \mathcal{O}_{X}(+1)^{\oplus 3} \oplus \mathcal{O}_{X}(+2)
$$

so that

$$
\operatorname{dim} H^{0}\left(N_{X}\right)=3 h^{0}\left(\mathcal{O}_{X}(+1)+h^{0}\left(\mathcal{O}_{X}(+2)\right) .\right.
$$


Now by $[\mathrm{Ku} 3,1.3]$ one has by Riemann-Roch, for the Fano 4 -fold $X$ of index 1 ,

$$
h^{0}\left(-m K_{X}\right)=1+\frac{m(m+1)}{24}\left(-K_{X}\right)^{2} c_{2}(X)+\frac{m^{2}(m+1)^{2}}{24}\left(-K_{X}\right)^{4}
$$

Since $X$ is a smooth 4 -fold of degree 28 in $\mathbf{P}^{11}$, where the class of its hyperplane section is $\left(-K_{X}\right)$, one has

$$
h^{0}\left(-K_{X}\right)=12,
$$

and so

$$
\left(-K_{X}\right)^{4}=28
$$

leading to

$$
12=1+\frac{1}{12}\left(-K_{X}\right)^{2} c_{2}(X)+\frac{28}{6},
$$

or $\left(-K_{X}\right)^{2} c_{2}(X)=76$, hence $h^{0}\left(-2 K_{X}\right)=62$. Finally,

$$
h^{0}\left(N_{X}\right)=3.12+62=98 .
$$

Now Prof. Peternell has called attention to the exact sequence

$$
0 \longrightarrow I_{X} \otimes T_{Y} \longrightarrow T_{Y} \longrightarrow T_{Y} \mid X \longrightarrow 0
$$

and this leads to a long exact cohomology sequence of which a part is

$$
\begin{aligned}
0 & \longrightarrow H^{0}\left(Y, T_{Y} \otimes I_{X}\right) \longrightarrow H^{0}\left(T_{Y}\right) \\
& \longrightarrow H^{0}\left(T_{Y} \mid X\right) \longrightarrow H^{1}\left(Y, T_{Y} \otimes I_{X}\right) \longrightarrow
\end{aligned}
$$

of which we now know that the second and last terms are $\{0\}$; namely,

$$
H^{i}\left(Y, T_{Y} \otimes I_{X}\right)=\{0\}, \quad i=0,1
$$

and hence

$$
h^{0}\left(T_{Y} \mid X\right)=h^{0}\left(T_{Y}\right)=\operatorname{dim} \operatorname{Aut}(Y)=35 .
$$

Now by $[\mathrm{Ku} 2,1.3 \mathrm{~b}]$ we have

$$
H^{1}\left(X, T_{Y} \mid X\right)=\{0\}
$$


so from the exact sequence

$$
\begin{aligned}
& 0 \longrightarrow H^{0}\left(T_{X}\right) \longrightarrow H^{0}\left(T_{Y} \mid X\right) \longrightarrow H^{0}\left(N_{X}\right) \\
& \longrightarrow H^{1}\left(T_{X}\right) \longrightarrow H^{1}\left(X, T_{Y} \mid X\right)=\{0\}
\end{aligned}
$$

and from $h^{1}\left(T_{X}\right)=63$ we obtain

$$
63=h^{0}\left(N_{X}\right)-h^{0}\left(T_{Y} \mid X\right)+h^{0}\left(T_{X}\right)=98-35+h^{0}\left(T_{X}\right) .
$$

Therefore, $h^{0}\left(T_{X}\right)=\operatorname{dim} H^{0}\left(T_{X}\right)=0$ as claimed earlier. Thus, by Theorem 6.4 on page 306 of Kodaira's Springer text [Ko], the family of $X$ is complete and effectively parametrized at all its points and the dimension of the base $\Delta$ of any complete, effectively parametrized complex analytic fiber system $\left(M_{\Delta}, \Delta, \varpi\right)$ with $X$ as one of its fibers, $X=\varpi^{-1}(\delta), \delta \in \Delta$, is $m(X)=63$. What remains open is whether this family has a global moduli space.

\section{References.}

[AA] Baily, W., A problem on an exceptional domain. In "Algebra and Analysis". Eds. Arslanov, Parshin, Shafarevich. Copyright: Walter de Gruyter, 1996.

[Bor1] Borcea, C., Families of smooth hypersurfaces on certain compact homogeneous complex manifolds. Math. Proc. Camb. Phil. Soc. 93 (1983), 315-321.

[Bor2] Borcea, C., Smooth global complete intersections in certain compact homogeneous complex manifolds. J. reine ang. Mathematik 344 (1983), 65-70.

[Bot] Bott, R., Homogeneous vector bundles. Ann. Math. 66 (1957), 203-248.

[Ko] Kodaira, K., Complex Manifolds and Deformation of Complex Structures. Grundlehr. d. math. Wissenschaften 283 Springer-Verlag, 1986.

[Ko-Sp] Kodaira, K., Spencer, D. C., On Deformations of Complex Analytic Structures I-II, III. Ann. of Math. 67 (1958), 328-466, 71 (1960), 4376 .

[Ku1] Küchle, O., On Fano 4-folds of index 1 and homogeneous vector bundles over Grassmannians. Math. Z. 218 (1995), 563-575.

[Ku2] Küchle, O., Some properties of Fano manifolds that are zeros of sections in homogeneous vector bundles over grassmannians. Pacific. J. Math. 175 (1996), 117-125. 
[Ku3] Küchle, O., Some remarks and problems concerning the geography of Fano 4-folds of index and picard number one. Questiones Mathematicae 20 (1997), 1-13.

[We] Wehler, J., Deformation of varieties defined by sections in homogeneous vector bundles. Math. Ann. 268 (1984), 519-532.

Recibido: 20 de junio de 2.001

Walter L. Baily, Jr.

Department of Mathematics

University of Chicago

5734 University Ave.

Chicago, Illinois 60637-1546, U.S.A.

baily@math . uchicago . edu 